\title{
Aurélie NÉVOT, «Comme le sel, je suis le cours de l'eau», le chamanisme à écriture des Yi du Yunnan (Chine)
}

Nanterre, Société d'ethnologie, 2008, 317 p.

Benoît Vermander

\section{QpenEdition}

\section{Journals}

Édition électronique

URL : http://journals.openedition.org/assr/21626

DOI : 10.4000/assr.21626

ISSN : $1777-5825$

Éditeur

Éditions de l'EHESS

Édition imprimée

Date de publication : 31 décembre 2009

Pagination : 75-342

ISBN : 978-2-7132-2218-4

ISSN : 0335-5985

\section{Référence électronique}

Benoît Vermander, «Aurélie NÉvot, «Comme le sel, je suis le cours de l'eau», le chamanisme à écriture des Yi du Yunnan (Chine) », Archives de sciences sociales des religions [En ligne], 148 | octobredécembre 2009, document 148-96, mis en ligne le 27 janvier 2010, consulté le 21 septembre 2020. URL : http://journals.openedition.org/assr/21626 ; DOI : https://doi.org/10.4000/assr.21626

Ce document a été généré automatiquement le 21 septembre 2020

(c) Archives de sciences sociales des religions 


\title{
Aurélie NÉVOT, «Comme le sel, je suis le cours de l'eau», le chamanisme à écriture des Yi du Yunnan (Chine)
}

Nanterre, Société d'ethnologie, 2008, 317 p.

\author{
Benoît Vermander
}

\section{RÉFÉRENCE}

Aurélie NÉVOT, «Comme le sel, je suis le cours de l'eau», le chamanisme à écriture des Yi du Yunnan (Chine), Nanterre, Société d'ethnologie, 2008, 317 p.

1 L'ouvrage se donne pour ambition d'étudier «la religion chamanique des Nipa», un petit groupe culturel et linguistique rattaché par l'État chinois à la «nationalité» yi. Les Yi constituent l'une des cinquante-cinq minorités nationales officiellement reconnues en Chine continentale. Le recensement ethnique de 1953-1956 a amalgamé sous ce terme des groupes établis dans les provinces de Yunnan, Sichuan (préfecture de Liangshan), et Guizhou. Ce sont près de huit millions de personnes qui sont identifiées comme Yi, des populations que divisent l'usage de six langues, toutes tibéto-birmanes (on ne saurait parler de «grands dialectes» comme le fait l'auteur p.21, reprenant un terme de linguistes chinois - il s'agit bien de langues, parfois très différenciées) et vingt-six dialectes pour le moins - certaines de ces langues restant dominantes dans les zones où elles sont parlées, d'autres étant en voie d'extinction - ainsi que des conditions matérielles, une mémoire historique et des traditions rituelles et culturelles des plus variées. Ne pas en conclure que le terme Yi n'est qu'un mot vide de contenu. Non seulement la complexité de l'histoire des populations non han du sud-ouest de la Chine rend tout regroupement à la fois aléatoire et pourtant justifiable, mais encore l'usage d'une catégorie unique a d'ores et déjà créé une conscience commune, mise en avant par nombre d'intellectuels issus des groupes en question. Quoi qu'il en soit, les 
Nipa (ou Sani) s'inscrivent dans cet ensemble composite. L'ouvrage ne spécifie pas la taille de la population étudiée.

Une introduction spécifie les principales notions dont l'ouvrage fait usage: délimitation du groupe étudié; "chamanisme à écriture»; culte fédéral officiel et culte local qui fonde l'identité villageoise. La première partie s'essaie à ressaisir la mémoire nipa et les origines du groupe, puis en étudie l'organisation villageoise. L'auteur voit dans les «maitres de la psalmodie» (bimo) un vecteur porteur de cette identité, et examine à cette lumière leur écriture rituelle et la transmission du savoir et de la fonction. La deuxième partie confronte l'organisation villageoise aux contraintes et requêtes de l'État chinois, prenant pour exemple l'uniformisation des écritures et la création d'un culte fédéral «orchestré» par les autorités politiques. Les troisième, quatrième et cinquième parties pourraient être aisément regroupées, puisqu'elles sont consacrées à l'étude d'un même rituel villageois, tenu à l'approche de l'hiver. Ordonnancement de l'univers, rapport aux ancêtres et narration des origines constituent les axes organisateurs de cette étude de cas: les pratiques et textes rituels peuvent être lus comme une narration des origines, redoublée par la spatialisation mythique du territoire. La spatialisation opère "par moitiés», l'une des expressions de cette opération formelle se trouvant dans le partage par deux villages d'ancêtres fondateurs communs, lequel partage établit entre eux une relation faite tout à la fois de solidarité et de rivalité. On peut discerner là un trait ordonnateur de l'ancienne chefferie nipa.

3 La conclusion résume bien les thèses principales de l'ouvrage: le contrôle des bimo et de leur écriture est un enjeu de pouvoir, que le gouvernement sait utiliser à son profit, en même temps qu'il met en place la nouvelle économie touristique de la province. Cette instrumentalisation n'empêche pas les bimo de conserver un rôle important dans la survie de l'ordre hiérarchique local traditionnel, exemplifié par la tenue de rituels saisonniers. Cette ambivalence entre société globale et ordre local est à terme créatrice d'une nouvelle «entité politique» (p.287), processus dont témoignent les transformations de l'écriture rituelle.

4 L'ouvrage a plusieurs mérites, dont le principal est de se mesurer avec un matériau au maniement particulièrement malaisé: les groupes yi du Yunnan ont été progressivement «hanisés» (sinisés) depuis plusieurs siècles; si l'on peut, de fait, les identifier par des langues et des mythes partagés, les frontières qui les distinguent les uns des autres, comme la nature de leur interaction avec la culture et le pouvoir han, soulèvent des interrogations permanentes, qui n'admettent que des réponses hésitantes et partielles. L'étude des groupes yi du Yunnan se trouve ainsi compliquée par trois facteurs: la difficulté des distinctions intra-ethniques, essentielles pour la compréhension des systèmes d'alliance et des parcours de mémoire; la validité ou nonvalidité des références aux études portant sur la religion et la société han, dans le cas de sociétés fortement métissées; enfin, la domination des études yi par les recherches portant sur la préfecture de Liangshan: la quasi-indépendance de Liangshan jusqu'aux lendemains de la Libération en a fait, par contrecoup, le modèle d'une société yi mythiquement reconstruite, à l'aune de laquelle sont souvent évalués les autres groupes yi, soumis depuis bien plus longtemps à la domination politique han. Vue historique faussée, mais qui explique l'avantage relatif dont jouit Liangshan en termes d'études ethnographiques. Liangshan n'est pas le «centre» d'une culture yi unifiée dont les autres régions constitueraient la périphérie, offrant des variantes à rapporter à une matrice culturelle. L'étude détaillée d'un groupe «frontière» comme le sont les Nipas 
apporte donc une perspective comparatiste des plus intéressantes. Par ailleurs, l'ouvrage offre un excellent récit de la fabrication d'un rite «officiel» par les autorités fédérales, récit qui respecte l'ambivalence des situations de terrain, et montre bien la complicité/ rivalité entre représentants des minorités et pouvoir central. Le récit du rituel saisonnier rapporté dans les trois dernières parties est également riche, attentif et bien informé.

Plusieurs problèmes n'en limitent pas moins la portée du travail ici présenté: en premier lieu, l'ouvrage est fortement «a-historique», à peu près muet quant aux développements des deux derniers siècles qui ont déterminé les rapports existants entre société nipa et pouvoir han (alors même qu'il fait du rapport entre l'État et la société locale dans la période la plus récente un enjeu essentiel de la démonstration); il procède de ce fait à des «sauts» qui autorisent des conclusions pour le moins osées sur «l'origine» de la société nipa (pp.46-52). Problème corollaire: la littérature existante est traitée trop rapidement. Si les ouvrages de Stevan Harrell et autres sont indiqués en bibliographie, leurs résultats n'ont pas l'air d'être pris en compte: la lecture attentive de Ways of Being Ethnic in Southwest China (Seattle, University of Washington Press, 2001), notamment, aurait permis à l'auteure une compréhension bien plus fine des mécanismes d'interaction entre microgroupes ethniques comme entre minorités et populations han dans cette partie de la Chine. Les mythes utilisés (dont l'origine n'est pas toujours précisée) auraient alors été traités avec davantage de prudence. Durant les dernières années, les études conduites sur cette partie de la Chine du sud-ouest ont montré la variété des adaptations locales, la manière de tirer avantage d'une situation minoritaire ou au contraire de l'atténuer ou de la nier, la façon dont un individu ou une micro-communauté navigue avec aisance dans un réseau de multi-appartenances. Elles ont aussi illustré une relative autonomie de la catégorie d'identité ethnique, laquelle est en partie indépendante d'une revendication proprement politique ou même d'une spécificité culturelle ou linguistique - il s'agit d'une autonomie de la mémoire et du projet pourrait-on dire, la spécificité de la mémoire aidant à construire un modèle propre d'adaptation et de développement local. Contrairement aux régions frontalières de la Chine, la situation des régions situées à la limite du Sichuan et du Yunnan est marquée par le compromis, l'adaptation, une façon de vivre son ethnicité qui permet d'en tirer des avantages résiduels. L'ouvrage reste en deçà de ces recherches, et, de ce fait, peine parfois à tisser en un tout convaincant ses analyses sur les rituels et sur les questions identitaires. On s'étonne notamment de l'absence de toute mention du livre d'Eric Mueggler (The Age of Wild Ghosts, Memory, Violence and Place in Southwest China, Berkeley, University of California Press, 2001) qui porte sur une population presque contiguë de celle ici étudiée et se mesure exactement aux mêmes questions: identité, mémoire sociale et rituels. Or, l'ouvrage de Mueggler aurait sans nul doute considérablement aidé l'auteure à sortir d'une perspective parcellaire et a-historique pour bâtir peut-être un récit et un système d'interprétation qui prenne en compte la genèse et la transformation des rituels dans un processus historique continu, où passé proche et récits des origines se rejoignent et tissent leurs fils. The Age of Wild Ghosts a renouvelé la façon d'aborder une microrégion des marches chinoises (Zhizuo, dans la préfecture autonome de Chuxiong, au nord du Yunnan, est une zone toute voisine de celle étudiée par Aurélie Névot et traite d'un groupe cousin). Que l'on compare, par exemple, la description de l'institution du ts'ici, faite par Mueggler, à celle du midjeoma par A.Névot (pp.126-130: le point n'est pas ici de reprocher à l'auteur l'absence de tel ou tel ouvrage dans sa bibliographie, reproche toujours trop facile, mais plutôt de 
comparer deux méthodes d'approche, dont l'une peine à insérer l'approche des rituels dans une perspective socio-historique cohérente à une autre qui, malgré ses limites, a choisi de développer de bout en bout une métaphore organisatrice - la métaphore corporelle dans ce cas précis), avec une précision dans les détails qui peu à peu la rend crédible et opérationnelle. La référence au livre de Mueggler est pertinente pour une autre raison: Mueggler a commencé par étudier des rituels habituellement classés comme religieux, avant de ne les envisager qu'en fonction de leur rapport à une identité personnelle, culturelle et sociale en perpétuelle reformulation. Résultat d'une évolution notable en anthropologie (et pas seulement dans l'anthropologie américaine), le mot «religieux» est absent de son livre. On assiste ainsi à une séparation de plus en plus nette entre une littérature anthropologique, pour laquelle il n'est pas d'autonomie ni même d'existence du fait religieux en soi, et des «sciences religieuses» à l'objet mal défini mais de plus en plus englobant, pour lesquelles tout, en fin de compte, devient religieux. On peut à l'inverse faire le constat de la persistance d'un système de croyances qui détermine et limite l'utilisation du croyable disponible aux fins d'affirmer une identité personnelle, politique ou culturelle. C'est le choix que semble effectuer Aurélie Névot, quoique de façon beaucoup trop implicite.

6 Autre point: la précision du vocabulaire et des notions est parfois grande, parfois soudainement négligée. Ainsi, pour caractériser le groupe étudié il est question à la même page (p.28) du "clan ni» et du "peuple ni» (Les hésitations continuelles sur l'emploi du terme de clan sont dommageable. Pour l'étude de ce continuum que sont les ethnies yi, la prévalence ou non d'une structure clanique est un élément discriminant essentiel). Les traductions sont souvent belles mais parfois un peu «tirées» et pas toujours justifiées, elles peinent à faire justice à la concision des récitations rituelles (voir pp.189-196, et voir en parallèle les pages sur le rythme de marche en montagne de la poésie rituelle in Mueggler, pp.238-247). Ou encore: parler d'«écritures secrètes» me semble une exagération stylistique: les diverses écritures yi étaient peu apprises et usitées en dehors du contexte rituel, mais on trouve aussi dans ces écritures des livres à caractère historique (généalogies, récits, chroniques de guerre), les traités d'astronomie, de médecine, de géographie ou de morale (certains diffusés par de riches Yi au début $d u x^{e}$ siècle). Des nzymo savaient certainement lire et écrire. Le fait que l'écriture fût peu usitée et que son efficace dépende toujours du statut de qui procède à sa lecture publique ne la rend pas «secrète» à proprement parler. Dernier exemple: on est surpris de l'imprécision du vocabulaire utilisé pour parler des «esprits» invoqués dans les rituels et sacrifices, et notamment de l'absence d'expressions éventuellement utilisées pour caractériser les «démons» (quelle que soit la façon dont on désigne les esprits maléfiques), alors que leur conjuration occupe habituellement une place prépondérante dans les pratiques et croyances religieuses des groupes yi et avoisinants. Si d'aventure tel n'était pas le cas chez les Nipa (ce dont je doute), l'étrangeté du phénomène mériterait discussion. La fixation sur les procédures à employer pour assurer le passage des défunts au statut d'esprit ancestral bienveillant (le danger étant grand de voir les morts devenir démons affamés) représente une constante de la littérature et des résultats bruts des enquêtes en territoire yi. Que les résultats de la recherche d'Aurélie Névot ne l'orientent pas dans ce sens serait en soi tout à fait légitime - à condition qu'une éventuelle «exception nipa» trouve explication ou que le paradigme alternatif soit clairement articulé.

Or, la description du grand rituel relaté dans les parties trois à cinq, si elle abonde en détails, souffre d'un certain manque d'unité conceptuelle. Les emprunts sont un peu 
hétéroclites. On est gêné par les références répétées à Marcel Granet et à «l'antiquité chinoise» dans le cadre d'une étude portant sur une population dont la langue et la culture originelles appartiennent au système tibéto-birman. Certes, la «hanisation» progressive des Nipa peut justifier l'emploi de catégories développées dans un autre contexte, mais, là encore, à condition d'esquisser une séquence historique. De même les références à Strickmann, Stein ou Malamoud sont intéressantes, parfois judicieuses, mais la transposition culturelle opérée n'est jamais justifiée, et ces références n'organisent pas un système global d'interprétation. À l'image du discours tenu par de nombreux auteurs travaillant dans des institutions académiques du Yunnan, Aurélie Névot rapproche fortement la religion des sous-groupes yi des pratiques taoïstes (même si ces rapprochements restent souvent implicites), mais nombre des analogies établies entre ces deux systèmes n'ont jamais été vraiment justifiées ni historiquement ni en termes d'une synthèse cosmologique vraiment convaincante; ils servent plutôt à inscrire les groupes yi dans «la vision chinoise» classique (pour des auteurs yi, le rapprochement entre religions yi et taoïsme permet aussi de mettre en valeur la contribution de leur peuple à la civilisation chinoise, et à relever ainsi leur statut propre) - cela même à quoi A.Névot voudrait pourtant échapper.

Enfin, l'emploi même des catégories de la «religion chinoise» telle que systématisé par les sociologues et sinologues de la première moitié $\mathrm{du} \mathrm{xx}^{\mathrm{e}}$ siècle, irait plutôt à l'encontre du projet initial du livre, à savoir la caractérisation d'un «chamanisme à écriture». Si le terme est explicité au début du livre (pp.24-26), la suite de l'ouvrage ne convainc pas vraiment de sa pertinence - ni, du reste, de sa non-pertinence: un point néanmoins pour apprécier la pertinence finale du concept: pour justifier son emploi du terme de "chamane» plutôt que celui (de fait contestable) de «prêtre devin», Aurélie Névot affirme qu'il ne faut pas surestimer la fonction divinatoire du bimo (p.26). L'observation porte plutôt à penser que, jusqu'à présent, les études n'ont pas accordé aux pratiques divinatoires l'importance qu'elles méritent pour la compréhension de la cosmologie et des croyances des groupes yi et avoisinants. À la fin de la lecture on retire l'impression que les pratiques des bimo nipa peuvent ou ne peuvent pas être qualifiées de «chamaniques», mais que cette qualification éventuelle reste presque annexe dans la description des phénomènes rapportés, lesquels se mesurent plutôt avec la mémoire d'un lieu et d'une identité menacés. L'ajout «à écriture» est rapporté à la caractérisation de cette écriture comme «rituelle, secrète et initiatique (...) en relation avec la transe», caractérisation qui, je l'ai dit, me semble aller plus loin que ce que l'observation permet d'affirmer.

9 Les réserves exprimées à l'instant ne sauraient dissimuler le fait que l'enquête ici rapportée porte sur un groupe et une région encore peu observés, est riche en hypothèses et en observations, et ouvre d'intéressantes perspectives comparatistes tant il s'agit désormais de regarder les groupes ethniques du sud-ouest chinois comme un ensemble souple et ramifié, un ensemble dépourvu d'un centre ou d'un point de référence majeur mais qui s'appréhende dans la multiplicité des interactions et leur perpétuelle recomposition. Il faut néanmoins interpréter le matériau et les assertions de l'ouvrage avec une certaine prudence, et prendre parfois quelque distance avec des thèses qui ne sont pas toujours illustrées ni prouvées comme on voudrait qu'elles le soient. 